\title{
Valor nutricional da polpa de açaí (Euterpe oleracea Mart) liofilizada
}

\author{
Ellen Mayra da Silva MENEZES ${ }^{1}$, Amanda Thiele TORRES², Armando Ubirajara Sabaa SRUR ${ }^{3}$
}

\begin{abstract}
RESUMO
O Brasil é o maior produtor, consumidor e exportador da bebida açaí produzida a partir dos frutos do açaizeiro. Esta bebida ou a polpa de açaí são normalmente comercializadas a temperatura ambiente ou na forma congelada, levando à perdas nutricionais importantes. Este trabalho objetivou analisar alguns nutrientes da polpa de açaí liofilizada. Os resultados de determinaçōes analíticas mostraram que esse produto na forma de pó é um alimento altamente calórico, 489,39 Kcal/100 g de polpa liofilizada principalmente em função dos altos conteúdos de lipídeos $(40,75 \%)$, dos quais 52,70\% representado pelo ácido oléico $\left(\mathrm{C}_{18: 1}\right)$ e 25,56\% pelo palmítico $\left(\mathrm{C}_{16 \cdot 0}\right)$. $\mathrm{O}$ teor de carboidratos totais foi de $42,53 \% \pm 3,56$ e o de proteínas foi de $8,13 \mathrm{~g} \pm 0,63$ por $100 \mathrm{~g}$ de açaí liofilizado. Na avaliação do perfil de minerais foi demonstrado que o potássio $(900 \mathrm{mg} / 100 \mathrm{~g}$ de polpa de açaí liofilizado) e o cálcio ( $330 \mathrm{mg} / 100 \mathrm{~g}$ de polpa de açá liofilizada) foram os minerais observados em maior abundância. O magnésio também apresentou concentrações importantes (124,4 mg em $100 \mathrm{~g}$ de polpa liofilizada), diferente do ferro (4,5 mg em $100 \mathrm{~g}$ de polpa liofilizada). Diante dos resultados obtidos na avaliação da composição nutricional da polpa de açaí liofilizada, é possível concluir que esse processo pode ser considerado como uma excelente alternativa de conservação dessa polpa devido a presença de importantes componentes nutricionais encontrados na mesma.
\end{abstract}

PALAVRAS-CHAVE: Açaí, Liofilização, Valor nutricional.

\section{Lyophilized açaí pulp (Euterpe oleracea, Mart.) nutritional value}

\begin{abstract}
Brazil is the biggest producer, consumer and exporter of açaí drink, made from the açaí palm’s fruit. This drink or açaí pulp is usually commercialized under room temperature or in a frozen way resulting in important nutritional losses. This study aimed to evaluate some nutrients from the lyophilized açaí pulp. Humidity, total solids, ash and total lipids values were analyzed using the AOAC method. Carbohydrates including fibers were calculated by difference and the total nitrogen values were investigated by AACC method. Mineral concentration was analyzed in a mass spectrophotometer and fats by methyl esters preparation identified in a gas chromatography. Results from analytical determinations showed that the powder product is a very caloric food, $489.39 \mathrm{Kcal} / 100 \mathrm{~g}$ lyophilized pulp, specially due to the high lipid contents (40.75\%), from which $52.70 \%$ are represented by oleic acid $\left(\mathrm{C}_{18: 1}\right)$ and $25.56 \%$ by the palmitic $\left(\mathrm{C}_{16: 0}\right)$. Total carbohydrate content was $42.53 \% \pm 3.56$ and the protein's was $8.13 \mathrm{~g} \pm 0.63 / 100 \mathrm{~g}$. Mineral profile evaluation demonstrated that the potassium $(900 \mathrm{mg} / 100 \mathrm{~g}$ lyophilized açaí) and the calcium $(330 \mathrm{mg} / 100 \mathrm{~g}$ lyophilized açaí) were the minerals observed in abundance. Magnesium has also shown important concentrations (124.4 mg/100 g lyophilized açaí), different from the iron ( $4.5 \mathrm{mg} / 100 \mathrm{~g}$ lyophilized açaí). Based on the results obtained on the nutritional composition evaluation of the lyophilized açaí pulp, it is possible to conclude that this process can be considered as an excellent alternative for preserving this pulp due to the important nutritional compounds found in it.
\end{abstract}

KEYWORDS: Brazilian berry (açaí), Freeze dryer, Nutritional value.

\footnotetext{
${ }^{1}$ Mestre em Ciência e Tecnologia de alimentos, CPGCTA/DTA/UFRRJ. E-mail: ellenmayra@hotmail.com

${ }^{2}$ Aluna de Nutrição, DNBE/IN/UFRJ. e-mail: amanda.thiele@ig.com.br

${ }^{3}$ Professor Adjunto, DNBE/IN/UFRJ. e-mail: sabaasrur@yahoo.com.br
} 


\section{INTRODUÇÃO}

$\mathrm{Na}$ rica floresta Amazônica, o açaizeiro (Euterpe oleracea, Mart.) destaca-se por ser a palmeira mais produtiva desse Estuário, tanto em frutos como em gêneros derivados da planta. O fruto, matéria-prima para a obtenção do suco de açaí, bebida símbolo do estado do Pará, é o principal produto oriundo da palmeira. O Brasil se posiciona como o maior produtor, consumidor e exportador desse produto (Menezes, 2005). Entre os estados produtores de açaí, Pará, Maranhão, Amapá, Acre e Rondônia são os mais valorizados pela obtenção do fruto, sendo o primeiro, responsável por $95 \%$ da produção de açaí, calculada em 100 a 180 mil litros/ dia em Belém (Homma \& Frazão, 2002; Oliveira et al., 2002; Mendes, 2003). Hoje sua expansão econômica, já atinge novos mercados no sudeste do país e alguns países da Europa, Estados Unidos, Japão e China (Souto, 2001; Silva, 2002).

A polpa desse fruto tem sido objeto de alguns estudos em função de seu valor nutritivo e sensorial (Rogez, 2000; Souto, 2001; Menezes, 2005), sendo inclusive considerada como um alimento nutracêutico face ao seu rico conteúdo de antocianinas, pigmentos hidrossolúveis responsáveis pela cor avermelhada do fruto (Iaderoza et al., 1992; Ozela et al., 1997; Bobbio et al., 2000; Menezes 2005). As antocianinas tornaramse conhecidas por suas diversas propriedades farmacológicas e propriedades medicinais, incluindo anticarcinogênica, antiinflamatória e antimicrobiana, prevenindo a oxidação de proteínas de baixa densidade (LDL), enfermidades cardiovasculares e doenças neurológicas (Kuskoski et al., 2002; Alasalvar et al., 2005).

Nas regiōes produtoras, o produto derivado do açaí, predominantemente, é a polpa, comercializado normalmente à temperatura ambiente quando é imediatamente consumida, ou após certo período de refrigeração. Quando se destina aos comércios distantes, a polpa é congelada (Rogez, 2000), porém essa técnica de conservação provoca danos irreversíveis ao alimento, como perdas vitamínicas, alteraçôes reológicas e de cor, que modificam as propriedades originais (Menezes, 2005). É altamente perecível e de fácil deterioração, a temperatura ambiente, sua durabilidade é de poucas horas e sob refrigeração, o tempo máximo de conservação é de 12 horas (Rogez, 2000; Souto, 2001, Alexandre et al., 2004). Os fatores responsáveis por essas modificaçôes são de natureza microbiana, enzimática e química, ocasionando reaçōes de oxidação, redução dos teores de antocianinas e despigmentação da polpa, alterando as características desse produto com conseqüente desvalorização sensorial e até mesmo nutricional. Novos métodos de conservação dessa polpa foram estudados como a desidratação (Melo et al., 1998), irradiação (Souto, 2001) e emprego da alta pressão hidrostática (Menezes, 2005) visando prolongar a vida de prateleira e preservando as características originais do produto.
O objetivo deste trabalho foi avaliar o valor nutricional da polpa de açaí após sofrer liofilização.

\section{MATERIAL E MÉTODOS}

Polpa de açaí, tipo grosso (DOU, 2000) foi adquirida em estabelecimento comercial localizado em Belém - PA devidamente registrado no MAPA. A polpa obtida foi embalada em sacos plásticos de $1 \mathrm{~kg}$ e acondicionada em caixas de isopor contendo pedaços de gelo. No mesmo dia, foi transportada, por via aérea, para o Rio de Janeiro. A polpa chegou ao laboratório do DNBE/IN da UFRJ cerca de 12 $\mathrm{h}$ após ser produzida. No mesmo dia, a embalagem original foi removida e depositada em bandeja de aço inoxidável do liofilizador da marca LABCONCO, modelo Freeze dryer 8 que foi hermeticamente fechado e acionado o sistema de congelamento onde toda massa atingiu $-40^{\circ} \mathrm{C}$. Em seguida, o sistema de frio foi interrompido e o de vácuo foi acionado para que parte da água livre da polpa fosse sublimada. $\mathrm{O}$ processamento foi realizado em triplicata e durou em média 12 horas.

A polpa liofilizada foi acondicionada em sacos de polietileno com revestimento de folha de alumínio para impedir a passagem da luz, sendo fechados por pressão e armazenados a temperatura ambiente até a realização das análises.

$\mathrm{O}$ teor de umidade foi determinado em estufa a $105^{\circ} \mathrm{C}$ até peso constante segundo a AOAC (2000), sendo o resultado expresso em g de água/ $100 \mathrm{~g}$ de polpa de açaí liofilizada.

O teor de sólidos totais ( $\mathrm{g}$ de sólidos totais/100 g de polpa de açaí liofilizada) foi determinado segundo AOAC (2000).

$\mathrm{O}$ teor de cinzas (g de cinzas/100 g de popa de açaí liofilizada) foi determinado em mufla a $550^{\circ} \mathrm{C}$, segundo a AOAC (2000).

A determinação de nitrogênio total ( $\mathrm{g}$ de nitrogênio/100 $\mathrm{g}$ de polpa de açaí liofilizada) foi baseada no método de Kjeldahl tradicional, segundo AACC (1995). A concentração de proteína bruta foi calculada pelo produto da quantidade de nitrogênio total (g) pelo fator de conversão 6,25 (IAL, 2005).

Os teores de lipídeos totais ( $\mathrm{g}$ de lipídeos/100 g de polpa de açaí liofilizada) foram determinados com éter etílico em extrator de Soxhlet, segundo AOAC (2000).

A quantificação de carboidratos, incluindo as fibras foi calculada por diferença entre 100 e o soma dos teores de umidade, cinzas, lipídeos totais e proteínas.

A concentração de minerais foi determinada por espectrofotometria de massa com plasma indutivamente acoplado no modo semiquantitativo, utilizando o equipamento ELAN 6000 da Perkin Elmer-Sciex. 
Os ácidos graxos do liofilizado foram preparados com ésteres metílicos (Hartmann \& Lago, 1973), sendo a determinação dos ácidos realizada em cromatógrafo a gás da HEWLWTT PAKARD, modelo HP5890.

\section{RESULTADOS E DISCUSSÃO}

Os valores encontrados nas análises de composição centesimal da polpa de açaí liofilizada são mostrados na Tabela 1 e apresenta valor altamente calórico, 489,39 Kcal/ $100 \mathrm{~g}$ de polpa liofilizada e diferente dos reportados na literatura, 265 Kcal e $247 \mathrm{Kcal}$ em $100 \mathrm{~g}$ de matéria seca de polpa (INCAP, 1961 apud Rogez, 2000; ENDEF, 1977, apud Rogez, 2000), provavelmente em função do tipo de açaí, já que o tipo grosso utilizado no presente trabalho contém a maior concentração de sólidos totais que apresentam maiores concentração de nutrientes calóricos.

É importante salientar que seu alto valor energético se dá principalmente pelo seu conteúdo de matéria graxa (40,75\%), que correspondeu a $74,94 \%$ das calorias contidas no alimento, ao passo que, apenas $34,76 \%$ equivalem à energia oriunda dos carboidratos. Outros autores como Rogez (2000) e Menezes (2005) encontraram percentuais de lipídeos semelhantes de 52,64 e 42,73 g de lipídeos por $100 \mathrm{~g}$ de matéria seca de polpa de açaí.

A quantidade de carboidratos, $42,53 \% \pm 3,56$, contida na polpa de açaí liofilizada (Tabela 1) pode ter sido mascarada pelo teor de fibras, que não fora analisado na composição centesimal e foi incluído no cálculo de açúcares totais. Estudos demonstram que o açaí possui teor de carboidratos relativamente baixo, não sendo considerado um alimento fonte de hidratos de carbono (Rogez, 2000). Apesar de não ser pesquisada a fração de fibras alimentares, a polpa de açaí contém quantidade de fibras bastante expressiva, como mostraram estudos de Motta (1946) e Chaves \& Pechnik (1945) apud Rogez (1996), que relataram percentuais entre $30-32 \%$ (base seca) e de Yuyama et al. (2004), que

Tabela 1 - Composição centesimal da polpa de açaí liofilizada.

\begin{tabular}{lll}
\hline Determinações & $\mathrm{g} / 100 \mathrm{~g}$ de polpa liofilizada & Desvios padrões \\
\hline Energia (Kcal) & 489,39 & - \\
\hline Umidade & 4,92 & 0,12 \\
Cinzas & 3,68 & 0,08 \\
Proteínas & 8,13 & 0,63 \\
Lipídeos totais & 40,75 & 2,75 \\
Carboídratos totais & 42,53 & 3,56 \\
e fibras & & \\
\hline
\end{tabular}

encontraram 7,7, 5,3 e 7,2\% (base úmida) em polpas de três diferentes localidades do Ecossistema Amazônico. Esses valores chamaram a atenção de outros, principalmente, por atingirem percentuais próximos ao da concentração de lipídeos, o mais importante componente desse produto.

Os resultados das análises de proteínas na polpa liofilizada foram, em média, de $8,13 \pm 0,63 \mathrm{~g}$ de proteínas por $100 \mathrm{~g}$ de açaí liofilizado, valores bem próximos de Almeida \& Valsechi (1966 apud Rogez, 2000), ENDEF (1977 apud Rogez, 2000) e Rogez (2000), com 9,6, 7,02 e 10,05 g de proteína por 100 $\mathrm{g}$ de matéria seca de polpa de açaí. O teor protéico encontrado em bebidas de açaí varia de 1,5\% a 13\% (Bezerra et al., 2002). Embora a fração protéica dessa polpa seja baixa (Oliveira $e t$ al., 2002), não deve ser desprezado, pois existe ainda carência de informações a respeito do perfil de aminoácidos que constituem essa fração.

Na Tabela 2 estão apresentadas concentrações médias dos ácidos graxos do óleo da polpa de açaí liofilizada. $\mathrm{O}$ maior teor recai sobre o ácido oléico $\left(\mathrm{C}_{18: 1}\right)$ com $52,70 \%$, seguido do palmítico $\left(\mathrm{C}_{16: 0}\right)$ com $25,56 \%$. Achados semelhantes foram observados por Rogez (1996b apud Rogez, 2000) em sucos de açaí da cidade de Belém, com $54,9 \%$ de $\mathrm{C}_{18: 1}$, e na polpa por Lubrano et al. (1994 apud Rogez) que detectaram em suas maiores concentrações $60 \%$ de ácido graxo oléico e $22 \%$ do palmítico. Yuyama et al. (2004) também observaram importantes percentuais desses dois óleos com 58,7\% e 23\%, respectivamente. Embora o $\mathrm{C}_{18: 1}$ seja de valor essencial, deve ser destacado a presença do ácido linoléico $\left(\mathrm{C}_{18: 2}\right)$, também de caráter essencial a alimentação humana, onde foi encontrado $0,95 \%$ (Tabela 2). O mesmo foi observado por Rogez (1996b apud Rogez, 2000) (1,1\%), Lubrano et al. (1994 apud Rogez, 2000) (traços) e Yuyama et al. (2004) (0,7\%). Apesar da importância nutricional observada, o perfil de ácidos graxos demonstra que a polpa de açaí está altamente sujeita a autoxidação, podendo ser um dos parâmetros responsável pela reduzida vida de prateleira desse produto.

Tabela 2 - Percentual médio (\%) dos ácidos graxos da fração lipídica da polpa de açaí liofilizada.

\begin{tabular}{ll}
\hline Ácidos graxos & Percentual (\%) médio \\
\hline Palmítico $\left(C_{16: 0}\right)$ & 25,56 \\
\hline Palmitolé $\left(C_{16: 1}\right)$ & 3,54 \\
\hline Esteárico $\left(C_{18: 0}\right)$ & 1,84 \\
\hline Olêico $\left(C_{18: 1}\right)$ & 52,7 \\
\hline Linolêico $\left(C_{18: 2}\right)$ & 0,95 \\
\hline
\end{tabular}

* médias das amostras 
Os resultados do perfil de minerais pode ser observado na Tabela 3. O potássio $(900 \mathrm{mg} / 100 \mathrm{~g}$ de polpa de açaí liofilizado) e o cálcio ( $330 \mathrm{mg} / 100 \mathrm{~g}$ de polpa de açá́ liofilizada) foram os minerais observados em maior abundância, com concentrações similares às encontradas por Rogez (2000), $990 \mathrm{mg}$ de potássio e $309 \mathrm{mg}$ de cálcio, em $100 \mathrm{~g}$ de matéria seca de polpas de açaí, e por Almeida \& Valsechi (1966 apud Rogez, 2000), $1185 \mathrm{mg}$ de potássio e $241 \mathrm{mg}$ de cálcio em $100 \mathrm{~g}$ de matéria seca de polpa de açaí. A polpa liofilizada é considerada uma rica fonte destes dois minerais. De acordo com a Ingestão Adequada (RDA, 2004) são recomendadas para indivíduos adultos saudáveis $4,7 \mathrm{~g}$ de potássio/dia, o que poderia ser $38,3 \%$ suprido com o consumo de $200 \mathrm{~g}$ de polpa de açaí liofilizada e esta mesma quantidade satisfaria em $66 \%$ os valores referenciados na Ingestão Adequada para o consumo de cálcio (1000 mg para adultos saudáveis) de acordo com a DRI (1997).

Quando analisado o conteúdo de macro e microminerais (Tabela 3), a polpa de açaí liofilizada também apresentou importante concentração do mineral magnésio com 124,4 mg em $100 \mathrm{~g}$ de polpa liofilizada. Sabe-se que esse mineral desempenha papel fundamental no organismo humano, atuante no metabolismo dos carboidratos, proteínas e lipídeos (Dutra-de-Oliveira \& Marchini, 1998). Embora a deficiência de magnésio seja de difícil ocorrência, recomenda-se a ingestão de cerca de 320 a $420 \mathrm{mg}$ por dia (DRI, 1997) o que seria

Tabela 3: Conteúdo de elementos minerais na polpa de açaí liofilizada.

\begin{tabular}{llll}
\hline Mineral & $\begin{array}{l}\text { mg/100 g de } \\
\text { polpa liofilizada }\end{array}$ & Mineral & $\begin{array}{l}\text { mg/100 g de } \\
\text { polpa liofilizada }\end{array}$ \\
\hline Sódio $(\mathrm{Na})$ & 28,5 & Selênio $(\mathrm{Se})$ & $<0,02$ \\
\hline Magnésio $(\mathrm{Mg})$ & 124,4 & Prata $(\mathrm{Ag})$ & $<0,0002$ \\
\hline Alumínio (Al) & 0,36 & Cádmio $(\mathrm{Cd})$ & $<0,0002$ \\
\hline Manganês $(\mathrm{Mn})$ & 10,71 & Bário (Ba) & 0,34 \\
\hline Cobalto (Co) & 0,009 & Mercúrio $(\mathrm{Hg})$ & $<0,01$ \\
\hline Níquel (Ni) & 0,28 & Chumbo (Pb) & 0,014 \\
\hline Cobre (Cu) & 2,15 & Tório (Th) & 0,002 \\
\hline Zinco (Zn) & 2,82 & Urânio (U) & $<0,0001$ \\
\hline Arsênio (As) & $<0,004$ & Potássio (K) & 900 \\
\hline Rubídio (Rb) & 5 & Estrôncio (Sr) & 0,79 \\
\hline Molibdênio (Mo) & 0,013 & Antimônio (Sb) & $<0,0002$ \\
\hline Fósforo (P) & 54,5 & Ferro (Fe) & 4,5 \\
\hline Cálcio (Ca) & 330 & & \\
\hline
\end{tabular}

atendido com o consumo de cerca de 260 a 340 g de açaí liofilizado. Quando comparado aos valores de magnésio encontrados por Almeida \& Valsechi (1966 apud Rogez, 2000) e Rogez (2000) em 100 g de matéria seca de polpa de açaí, achados semelhantes foram observados com $140 \mathrm{mg}$ e $178 \mathrm{mg}$ deste mineral, respectivamente.

Diferentemente do que coloca a crença popular, o açaí não pode ser considerado como uma boa fonte de ferro, pois além desse mineral se encontrar na forma férrica (ou não heme) contida somente em vegetais, esta é de menor biodisponibilidade no organismo (Anderson et al., 1998), e apresenta valores médios de 2,6 mg de ferro/100 g de matéria seca (Rogez, 2000; Yuyama et al., 2002; Yuyama et al., 2004), estando muito aquém do recomendado por dia pela RDA (2001): equivalente a $18 \mathrm{mg}$ para mulheres em fase entre $19 \mathrm{e}$ 50 anos, fora de gravidez e $8 \mathrm{mg}$ para homens na mesma faixa etária. A polpa de açaí liofilizada apresentou $4,5 \mathrm{mg}$ de ferro em $100 \mathrm{~g}$, mostrando que a ingestão de $200 \mathrm{~g}$ de polpa de açaí liofilizada seria suficiente para atender as recomendaçôes para adultos do sexo masculino, entretanto exigiria um consumo maior por parte das mulheres. Vale ressaltar que além das diferenças entre o ferro hemínico e não hemínico, a biodisponibilidade desse último é fortemente influenciada por vários outros componentes da dieta (Cozzolino, 1997).

Diante dos resultados obtidos na avaliação da composição nutricional da polpa de açaí liofilizada, é possível concluir que esse processo pode ser considerado como uma excelente alternativa de conservação dessa polpa devido a presença de importantes componentes nutricionais encontrados na mesma. É também um excelente alimento a ser incorporado nas refeiçóes de indivíduos com baixo peso, em especial a faixa etária infantil, por ser altamente calórico. Além disso, adultos e adolescentes também deveriam incluir a polpa de açaí liofilizada nos seus cardápios, principalmente por serem afetados pelos hábitos alimentares do século XXI, que normalmente compõem refeiçôes pobres em macronutrientes e em minerais. O consumo dessa polpa, rica em lipídeos essenciais e de boa qualidade nutricional, e em certos minerais como o cálcio e o potássio podem contribuir para garantir o crescimento e bom funcionamento do corpo humano em geral, uma vez que esses nutrientes participam de varias reaçôes metabólicas importantes no organismo. Entretanto a polpa não pode ser considerada como uma fonte de ferro, sugerindose uma complementação desse mineral na dieta com alimentos fonte de ferro de maior biodisponibilidade.

\section{BIBLIOGRAFIA CITADA}

AACC(AMERICANASSOCIATION OF CEREAL CHEMISTRY), 1995. Approved Methods. St Paul, Minnesota, USA.

Alasalvar, C.; Al-Farsi, M.; Quantick, P.C.; Shahidi, F.; Wiktorowicz. 2005. Effect of chill storage and modified atmosphere packing 
(MAP) on antioxidant activity, anthocyanins, carotenoids, phenolics and sensory quality of ready-to-eat shredded orange and purple carrots. Food Chemistry, 89:69-76.

Alexandre, D.; Cunha, R.L.; Hubinger, M.D., 2004. Conservação do açaí pela tecnologia de obstáculos. Ciência e Tecnologia de Alimentos, 24(1): 114-119.

Anderson, L.; Dibble, M.V.; Turkki, P.R.; Mitchell, H.S.; Rynbergen, H.J. 1998. Nutrição. 17 a ed. Cap. 6. São Paulo, SP. 737pp.

AOAC (ASSOCIATION OF OFFICIAL ANALYTICAL CHEMISTRY), 2000. Official Methods of Analysis, $17^{\text {th }}$ ed, Washington, D.C. USA.

Bezerra, V.S.; Nery, M.V.S.; Lobato, M.S.A. 2002. Avaliação das características químicas do vinho de açaí comercializado em Macapá e Santana (AP). In: XVIII CONGRESSO BRASILEIRO DE CIÊNCIA E TECNOLOGIA DE ALIMENTOS, 2002, Porto Alegre. Anais... Rio Grande do Sul: Sociedade Brasileira de Ciência e Tecnologia de Alimentos.

Bobbio, F.O.; Druzian, J.I.; Abrão, P.A.; Bobbio, P.A.; Fadelli, S. 2000. Identificação e quantificação das antocianinas do fruto do açaizeiro (Euterpe oleracea) Mart. Ciência e Tecnologia de Alimentos, 20(3):388-390.

Cozzolino, S.M.F. 1997. Biodisponibilidade de minerais. Revista de Nutrição da Puccamp. 10(2): 87-98.

DOU - Diário Oficial da República Federativa do Brasil, 2000. Regulamento Técnico Geral para fixação dos Padrōes de Identidade e Qualidade para polpa de fruta. Instrução Normativa $\mathrm{n}^{\circ} 01$, de 07 de janeiro de 2000. Diário Oficial de 10 de janeiro , Brasília, DF.

DRI. Dietary Reference Intakes, 2004. In: Dietary Reference Intakes for Water, Potassium, Sodium, Chloride and Sulfate. Washington, D. C.: Academic Press, 2004. 640pp. Disponível em www.nap. edu. Acesso em 30/03/2005.

DRI. Dietary Reference Intakes, 1997. In: Dietary Reference Intakes for Calcium, Phosphorous, Magnesium, Vitamin D, and Fluoride. Washington, D. C.: Academic Press, 1997. Disponível em www. nap.edu. Acesso em 30/03/2005.

Dutra-de-Oliveira, J.E.; Marchini, J.S. 1998. Ciências Nutricionais. Ed. Sarvier, São Paulo. 403pp.

Hartmann, L.; Lago, R.C.A., 1973. Rapid preparation of fatty acid methyl esters from lipidis. Laboratory Practices, 22: 475-476.

Homma, A.K.O; Frazão, D.A.C. 2002. O despertar da fruticultura amazônica. Fruticultura em Revista, Novembro: 27-31.

Iaderoza, M.; Baldini, I.S.D.; Bovi, M.L.A. 1992. Anthocyanins from Fruits of Açaí (Euterpe oleracea, Mart.). Tropical Science, 32: 41-46.

IAL (INSTITUTO ADOLFO LUTZ). 2005. Normas Analiticas do Instituto Adolfo Lutz. Vol. 1. Métodos químicos e físicos para análises de alimentos. São Paulo, SP, Brasil. 371pp.

Kuskoski, E.M.; Fett, P.; Asuero, A.G. 2002. Antocianos: un grupo de pigmentos naturales. Aislamiento, identificación y propriedades. Alimentaria, 2(61): 61-74.
Melo, C.F.M.; Barbosa, W.C.; Alves, S.M. 1998. Obtenção de açaí desidratado. EMBRAPA/CPATU. 56pp.

Menezes, E.M.S. 2005. Efeito da alta pressão hidrostática em polpa de açai pré-congelada (Euterpe oleracea, Mart.). Dissertação de Mestrado em Ciência e Tecnologia de Alimentos, Universidade Federal Rural do Rio de Janeiro, Seropédica, RJ. 83pp.

Mendes, E. 2003. Demanda pode tornar açaí raro e caro no Pará, O Liberal. 15/02/2003. Disponível em www.oliberal.com.br. Acesso em 20/01/2004.

Oliveira, M.S.P.; Carvalho, J.E.U.; Nascimento, W.L.O.; Müler, C.H. 2002. Cultivo do Açaizeiro para Produção de Frutos. Circular Técnica. n. 26. Embrapa Amazônia Oriental. Belém, Pará.

Ozela, E.F.; Stringheta, P.C.; LIMA, A.A.S.; Farias, M.I.T.; Santos, M.V. 1997. Estudo comparativo do teor de antocianinas presentes no açaí (Euterpe oleracea Mart.), nos períodos de safra e entresafra. Simpósio Latino Americano de Ciência de Alimentos, 2, Campinas, 1997. Resumos...Campinas, UNICAMP.

RDA. Recommended Dietary Allowances, 2001. In: Dietary Reference Intakes for Vitamin A, Vitamin K, Arsenic, Boron, Chromium, Copper, Iodine, Iron, Manganese, Molybdenum, Nickel, Silicon, Vadium, and Zinc. Washington, D.C.: National Academic of Sciences.

Rogez, H.; De Souza, J.N.S.; Nascimento, M.Q.; Buxant, R. 1996. Branqueamento dos frutos de açaí (Euterpe oleracea, Mart.) para diminuição da carga microbiana do suco. Anais da Associação Brasileira de Quimica, 45(4): 177-184.

Rogez, H. 2000. Açaí: Preparo, Composição e Melhoramento da Conservação. Ed. Universidade Federal do Pará - EDUPA, Belém, Pará. 360pp.

Silva, P.R. 2002. Novidades na Fruticultura Paraense. Fruticultura em Revista. Belém, Pará. Novembro: 27-31.

Souto, R.N.M. 2001. Uso da radiação $\gamma$, combinada à refrigeração, na conservação de polpa de açaí (Euterpe oleracea, Mart.). Dissertação de Mestrado em Ciência e Tecnologia de Alimentos, Universidade Federal Rural do Rio de Janeiro, Seropédica, RJ. 95pp.

Yuyama, L.K.O.; Rosa, R.D.; Aguiar, J.P.L.; Nagahama, D.; Alencar, F.H.; Yuyama, K.; Cordeiro W.O.; Marques, H.O. 2002. Açaí (Euterpe oleracea Mart.) e Camu-Camu (Myrciaria dubia (H.B.K.) Mc Vaugh) possuem ação anti anêmica? Acta Amazônica, 32(4): 625-633.

Yuyama,L.K.O.; Aguiar, J.P.L.; Melo, T.; Barros, S.E.; Filho, D.F.S.; Yuyama, K.; Favaro, D.I.T.; Vasconcellos, M.; Pimentel, S.A.; Badolato, E.S.G. 2004. Açaí (Euterpe oleracea Mart.): Qual o seu potencial nutricional? In: XVII Congresso Brasileiro de Fruticultura, 2004. Florianópolis. Anais .... Santa Catarina: Sociedade Brasileira de Fruticultura.

Recebido em 20/06/2006

Aceito em 13/03/2008 
